\title{
molecules
}

ISSN 1420-3049

www.mdpi.com/journal/molecules

Article

\section{Antioxidant Mechanism of Rutin on Hypoxia-Induced Pulmonary Arterial Cell Proliferation}

\section{Qian Li ${ }^{1, \dagger}$, Yanli Qiu ${ }^{1, \dagger}$, Min Mao ${ }^{2,3}$, Jinying Lv ${ }^{1}$, Lixin Zhang ${ }^{2,3}$, Shuzhen Li ${ }^{2,3}$, Xia Li ${ }^{1}$ and} Xiaodong Zheng ${ }^{3, *}$

1 Department of Pharmaceutical Analysis, College of Pharmacy, Harbin Medical University, Nangang District, Harbin 150081, China; E-Mails: liqian@ems.hrbmu.edu.cn (Q.L.); yingzi1314179@126.com (Y.Q.); m15645109321@163.com (J.L.); fengyucaihong78@sohu.com (X.L.)

2 Bio-pharmaceutical Key Laboratory of Harbin, Harbin Medical University, Harbin 150081, China; E-Mails: mmarine510704@hotmail.com (M.M.); muzqian@sina.com (L.Z.); yw198269@163.com (S.L.)

3 Department of Pathophysiology, Harbin Medical University-Daqing, Daqing 163319, China

$\dagger$ These authors contributed equally to this work.

* Author to whom correspondence should be addressed; E-Mail: xzheng2001@163.com; Tel./Fax: +86-459-815-3399.

External Editor: Derek J. McPhee

Received: 26 August 2014; in revised form: 28 September 2014 / Accepted: 9 October 2014 / Published: 18 November 2014

\begin{abstract}
Reactive oxygen species (ROS) are involved in the pathologic process of pulmonary arterial hypertension as either mediators or inducers. Rutin is a type of flavonoid which exhibits significant scavenging properties on oxygen radicals both in vitro and in vivo. In this study, we proposed that rutin attenuated hypoxia-induced pulmonary artery smooth muscle cell (PASMC) proliferation by scavenging ROS. Immunofluorescence data showed that rutin decreased the production of ROS, which was mainly generated through mitochondria and NADPH oxidase 4 (Nox4) in pulmonary artery endothelial cells (PAECs). Western blot results provided further evidence on rutin increasing expression of Nox 4 and hypoxia-inducible factor-1 $\alpha$ (HIF-1 $\alpha$ ). Moreover, cell cycle analysis by flow cytometry indicated that proliferation of PASMCs triggered by hypoxia was also repressed by rutin. However, $\mathrm{N}$-acetyl-L-cysteine (NAC), a scavenger of ROS, abolished or diminished the capability of rutin in repressing hypoxia-induced cell proliferation. These data suggest that rutin
\end{abstract}


shows a potential benefit against the development of hypoxic pulmonary arterial hypertension by inhibiting ROS, subsequently preventing hypoxia-induced PASMC proliferation.

Keywords: rutin; reactive oxygen species (ROS); NADPH oxidase 4; proliferation; hypoxia

\section{Introduction}

Hypoxic pulmonary arterial hypertension (HPH) is a progressive disorder characterized by endothelial dysfunction, smooth muscle proliferation, intimal and/or medial layer remodeling, and right ventricular failure [1,2]. Remodeling of pulmonary blood vessels results from the proliferation of pulmonary arterial smooth muscle cells (PASMCs) and pulmonary arterial endothelial cells (PAECs) migration [3,4]. Endothelial cells are generally recognized as the main regulators of vascular function. Imbalance between vasoconstriction and vasodilation is characterized by ECs dysfunction [5]. PAECs dysfunction is involved in the development of $\mathrm{HPH}$ by contribution to the disturbed migration and proliferation of PASMCs [6]. However, the exact causes of HPH are still under investigation.

It has been shown that increasing oxidative stress augments HPH [7], whereas reducing oxidative stress reverses HPH [8]. These data suggest oxidative stress is implicated in the development of HPH [9]. During hypoxic condition, reactive oxygen species (ROS) released from PAECs, subsequently diffuses to PASMCs and causes calcium influx, which leads to pulmonary artery constriction [10]. Furthermore, ROS, such as superoxide anions and hydrogen peroxide $\left(\mathrm{H}_{2} \mathrm{O}_{2}\right)$ serve as regulators in the vascular smooth muscle cells proliferation [11,12]. NADPH oxidase 4 (Nox4) is a major isoform of NADPH oxidase responsible for the production of ROS which functions as a signaling molecule in cell survival and normal function [13].

Several signal transduction pathways and transcription factors are activated that could be involved in PASMC proliferation [14,15]. Proliferating cell nuclear antigen (PCNA) plays an important role in nucleic acid metabolism acting as a component for repair and replication machinery, and is known as a molecular marker for proliferation [16,17]. A previous study by our group indicated PCNA expression serves as an indicator for PASMCs proliferation [18]. Animal studies revealed a role for hypoxia-inducible factor-1 (HIF-1) in the development of HPH $[19,20]$. The $\beta$ subunit of HIF-1 (HIF-1 $\beta$ ) is constitutively expressed, whereas the $\alpha$ subunit (HIF-1 $\alpha$ ) is typically not detectable under normoxic conditions. Moreover, accumulation of HIF-1 $\alpha$ protein was observed in the lungs of PAH patients. A molecule that targets HIF-1 may provide a therapeutic potential for HPH.

Rutin, 3-((6-O-(6-deoxy- $\alpha$-L-mannopyranosyl)- $\beta$-D-glucopyranosyl)oxy)-2-(3,4-dihy-droxyphenyl)5,7-dihydroxy-4H-1-benzopyran-4-one (shown in Figure 1A), is the glycoside between the flavonol quercetin and the disaccharide rutinoside. Rutin restrains the oxidative progress. Based on its significant scavenging properties on oxidizing species, rutin has been implemented in ROS-related diseases, such as gastric lesions and diabetes [21,22]. Besides, rutin demonstrates several pharmacological activities, including anti-inflammatory, anti-allergic and vasoactive properties. In a previous study, we showed rutin attenuated hypoxic pulmonary vasoconstriction [23]. However, whether rutin has an effect on the abnormal proliferation in PASMCs is still unclear. In this study, we examined the effect of rutin on (1) scavenged PAECs ROS production; mitochondrial Nox4, which is connected to ROS production [24]; 
(2) inhibition of the expression of PCNA; and (3) decreased HIF-1 $\alpha$ expression, which was activated as a result of cell adaptive response under hypoxia. The aim was to determine whether rutin has an adjuvant therapy effect on HPH.

\section{Results and Discussion}

\subsection{Rutin Abolished Mitochondrial and Nox4-Generated ROS Production}

The production of ROS was measured with CM- $\mathrm{H}_{2}$ DCFDA. Hypoxia increased the ROS production in PAECs, as indicated by the increased fluorescence intensity (Figure 1B; Green). Mitochondria serve as the main organelle of cell metabolism and as an important producer of ROS. Then we examined ROS production in mitochondria using mitosox. The enhanced red flurorescence intensity indicated hypoxia also increased the ROS production in mitochondria (Figure 1B; Red). As proposed, rutin abolished the abnormal rise of ROS triggered by hypoxia both in PAECs and mitochondria (Figure 1B). Surprisingly, pre-treatment with APO (apocynin, NADPH oxidases inhibitor) or RE (rotenone, Mitochondria inhibitor) attenuated rutin's effect of decreasing ROS production (Figure 1C). The results demonstrated that rutin abolished the production of ROS, generated through Nox4 and mitochondrial under hypoxic conditions in PAECs.

Figure 1. Immunofluorescence staining using $\mathrm{CM}-\mathrm{H}_{2}$ DCFDA or mitosox to evaluate the production of reactive oxygen species (ROS) in pulmonary artery endothelial cells (PAECs) and mitochondria. (A) Chemical structure of rutin. (B) The increased production of ROS in cells (Green) and mitochondria (Red) induced by hypoxia was reduced when treated with rutin. (C) Cells were pretreated with APO (apocynin, NADPH oxidases inhibitor), RE (rotenone, mitochondria inhibitor), showed the reverse effect of rutin on pulmonary endothelial cells, respectively. ROS was assessed by a laser-scanning confocal microscope after treatment by CM- $\mathrm{H}_{2}$ DCFDA for the detection of the whole cells (Green) and mitosox (Red) for the detection of mitochondria respectively. Scale bars were $100 \mu \mathrm{m}$. Nor indicates normoxia, Hyp indicates hypoxia, Ru: rutin.
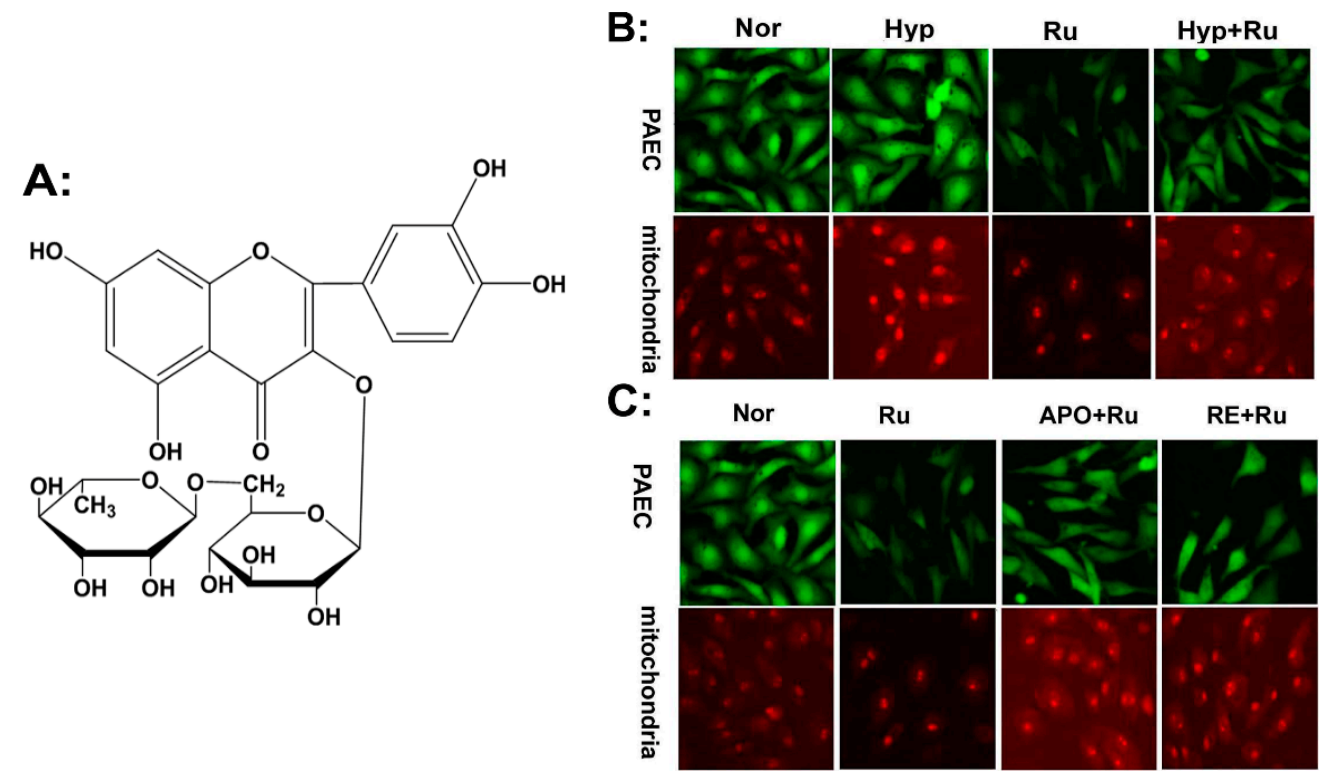


\subsection{Rutin Influenced the Expression of Nox4 under Hypoxia}

The decreased ROS production could be due to two factors, one less is produced, two more is scavenged. In order to examine which way was involved for the effect of rutin, the expression of Nox4 was elevated under hypoxic circumstances. Western blotting showed hypoxia increased Nox4 expression, and rutin decreased the up-regulation of Nox4 induced by hypoxia in both the PAECs (Figure 2A) and the PASMCs (Figure 2B). These data indicated rutin diminished ROS production by affecting Nox4 activity.

Figure 2. The regulative effect of rutin on the expression of NADPH oxidase 4 (Nox4) under hypoxia was evaluated by Western Blot. Under hypoxia, the activity of Nox 4 was decreased when treated with rutin, or $N$-acetyl-L-cysteine (NAC) $(5 \mu \mathrm{mol} / \mathrm{L})$ suppressed the effect of rutin evidently in PAECs (A) and pulmonary arterial smooth muscle cells (PASMCs). (B) All of the values were denoted as mean $\pm \mathrm{SD} ;{ }^{*} p<0.05$, versus normal; ${ }^{*} p<0.05$, versus Hyp. Nor indicates normoxia, Hyp indicates hypoxia.
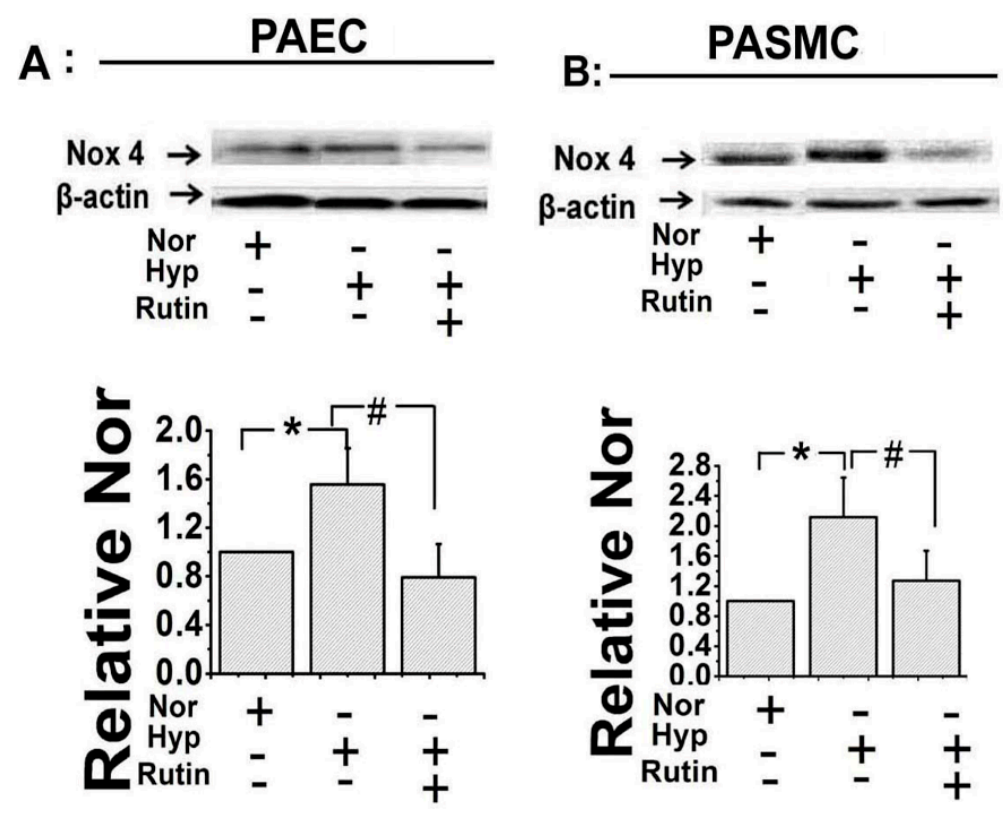

\subsection{Rutin Inhibited Hypoxia-Stimulated PAECs Migration and PASMCs Proliferation}

In order to examine the effect of rutin on PAECs migration under hypoxia, we performed a PAECs scratch-wound assay. The results showed that hypoxia significantly promoted PAECs migration, as shown in Figure 3A, rutin inhibited the hypoxia-trigged PAECs migration. Consistent with the results in Figure 1C, scavenged ROS by $N$-acetyl-L-cysteine (NAC, $5 \mu \mathrm{mol} / \mathrm{L}$ ) abolished the effect of rutin on inhibition of PAECs migration.

Hypoxia induced PASMCs proliferation is a character of hypoxia induced pulmonary hypertension. So we then examined whether rutin protected HPH through inhibiting PASMCs proliferation. Firstly, cell viability was measured by MTT, as shown in Figure 3B, hypoxia increased cell viability, and the effect was inhibited by rutin. Consistent with the PAECs migration data, pre-treatment with NAC, diminished the effect of rutin. Secondly, 5-bromodeoxyuridine incorporation assay showed a similar result, hypoxia increased 5-bromodeoxyuridine incorporation in PASMCs, the increasing effect was 
suppressed by rutin, whereas it was significantly attenuated in the presence of NAC (Figure 3C). Thirdly, PCNA, a symbol protein of cell proliferation and further remodeling of PA during the pathologic process in PAH was detected [17,25]. Hypoxia significantly increased the expression of PCNA in both the PAECs and the PASMCs (Figure 4). However, rutin reduced the hypoxia-induced upregulation expression of PCNA, whereas the inhibition effect was abolished by NAC (Figure 4). These results indicated that rutin inhibited hypoxia-induced PAECs migration and PASMCs proliferation, which may be through a ROS-dependent pathway.

Figure 3. Rutin blocked PAECs migration and PASMCs proliferation under hypoxia by regulating ROS. (A) The effect of hypoxia $(8 \mathrm{~h})$ on cell migration was blocked by rutin $(n=3)$, which was attenuated by NAC. Scale bars are $100 \mu \mathrm{m}$. (B) Cell viability was measured by MTT, the results were consistent with scratch-wound assay, rutin abolished hypoxia-increasing PASMCs viability, which was blocked with NAC. (C) NAC (ROS scavenger, $5 \mu \mathrm{mol} / \mathrm{L}$ ) increased 5-bromodeoxyuridine incorporation compared with the rutin group under hypoxia. All of the values were denoted as mean $\pm \mathrm{SD}$; ${ }^{*} p<0.05$, ** $p<0.01$, versus normal; \# $p<0.05,{ }^{\# \#} p<0.01$ versus Hyp, $n=3$. Nor indicates normoxia, Hyp indicates hypoxia.

A:
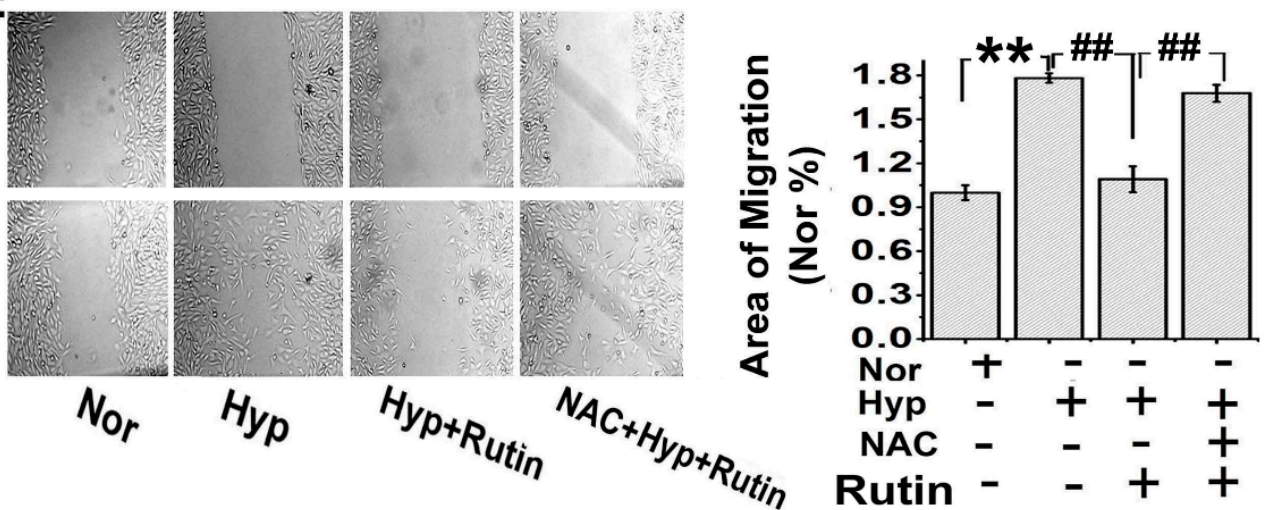

B:

C:
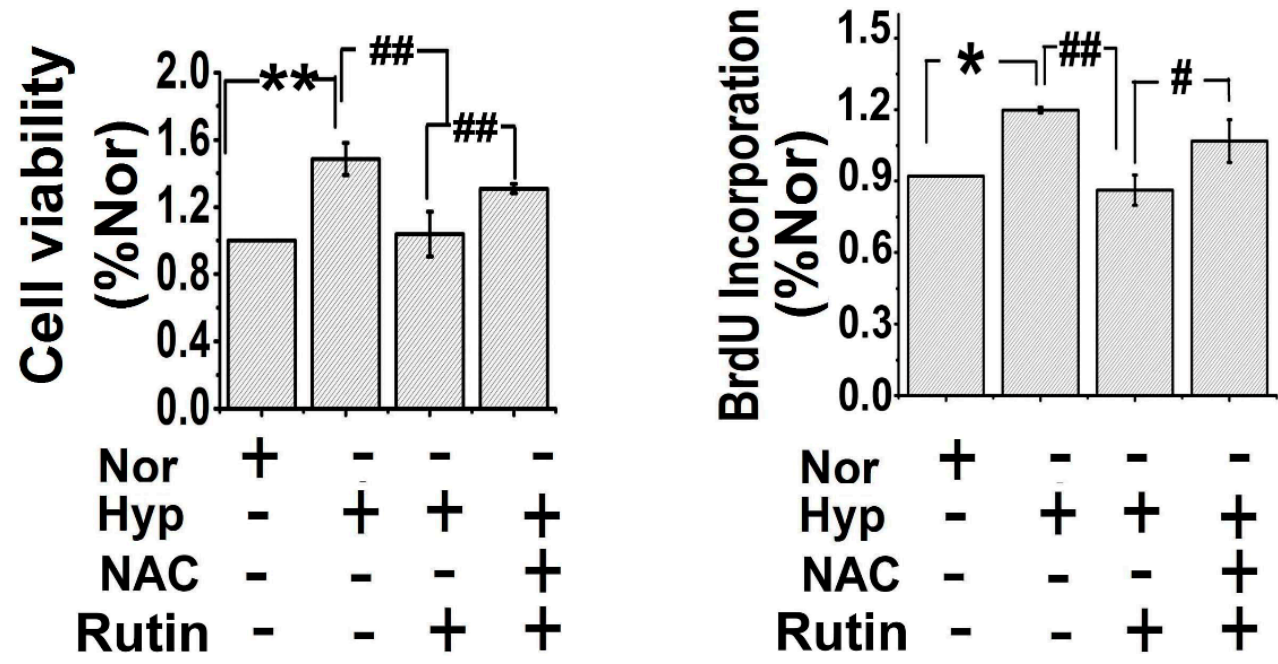
Figure 4. Rutin reversed the increased expression of proliferating cell nuclear antigen (PCNA) during hypoxia. Evaluation of the expression of PCNA in PAECs (A) and PASMCs (B) by Western Blot. The abnormal increased activity of PCNA induced by hypoxia was alleviated by rutin. All of the values were denoted as mean $\pm \mathrm{SD} ;{ }^{*} p<0.05$, versus normal; \#\# $p<0.01$ versus Hyp, $n=3$. Nor indicates normoxia, Hyp indicates hypoxia.
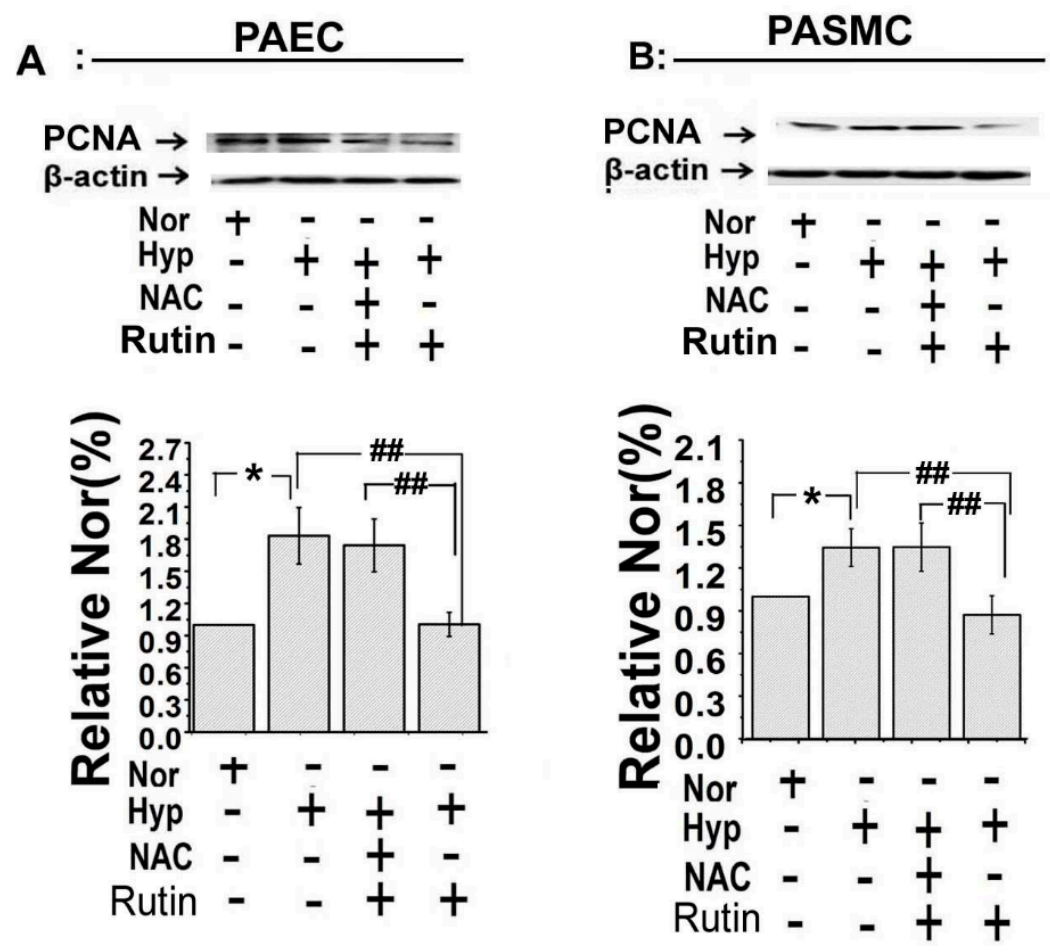

\subsection{Effect of Rutin on Hypoxia-Induced ROS on Cell Cycle Progression and Microtubule} Dynamic Stability

In order to confirm rutin regulated PASMCs proliferation process, we measured the cell cycle progression by flow cytometry, as shown in Figure 5A; hypoxia promoted PASMCs transit into S plus $\mathrm{G}_{2} / \mathrm{M}$ phases, rutin was able to repress the cell cycle progression whereas rutin's effects were attenuated with scavenged ROS by NAC (Figure 5A). Moreover, hypoxia induced the expression of housekeeper protein $\alpha$-tubulin in the nucleus of PASMCs (Figure 5B), which may promote mitotic spindle formation, and then contribute to PASMCs proliferation [26]. Rutin reversed this increasing expression of $\alpha$-tubulin, and the reversed effect was attenuated after scavenging ROS with NAC.

\subsection{The Upregulated Expression of HIF-1 $\alpha$ Induced by Hypoxia Was Depressed by Rutin}

The expression of HIF-1 $\alpha$ was linked to the abnormal proliferation of pulmonary arterial cells and further influenced the metabolism of mitochondria [27]. We detected the effect of rutin on the expression of HIF- $1 \alpha$ in both PASMCs and PAECs. Consistent with the PCNA expression, the expression of HIF-1 $\alpha$ was enhanced by hypoxia (Figure 6A), the upregulation was inhibited by rutin. Scavenged ROS by NAC attenuated rutin's effect. These data suggested that rutin inhibited the expression of HIF-1 $\alpha$ by reducing the production of ROS. 
Figure 5. The influence of rutin on the activity of PASMCs was detected with flow cytometry and the expression of $\alpha$-tubulin was recorded with immunohistochemistry. (A) Hypoxia promoted PASMCs cycle progression and increased the cell number in S plus G2/M phases, which was suppressed in the presence of rutin. The effect of rutin on hypoxia-induced enhanced cycle progression was eliminated with scavenged ROS by NAC. (B) The expression of $\alpha$-tubulin in PASMCs was upregulated under hypoxia which was blocked by rutin, and the role of rutin was eliminated in the presence of NAC (ROS scavenger, $5 \mu \mathrm{mol} / \mathrm{L}$ ). Scale bars were $100 \mu \mathrm{m}$. All of the values were denoted as mean $\pm \mathrm{SD} ; * * p<0.01$, versus normal; ${ }^{\#} p<0.01$, versus Hyp. $n=3$. Nor indicates normoxia, Hyp indicates hypoxia.

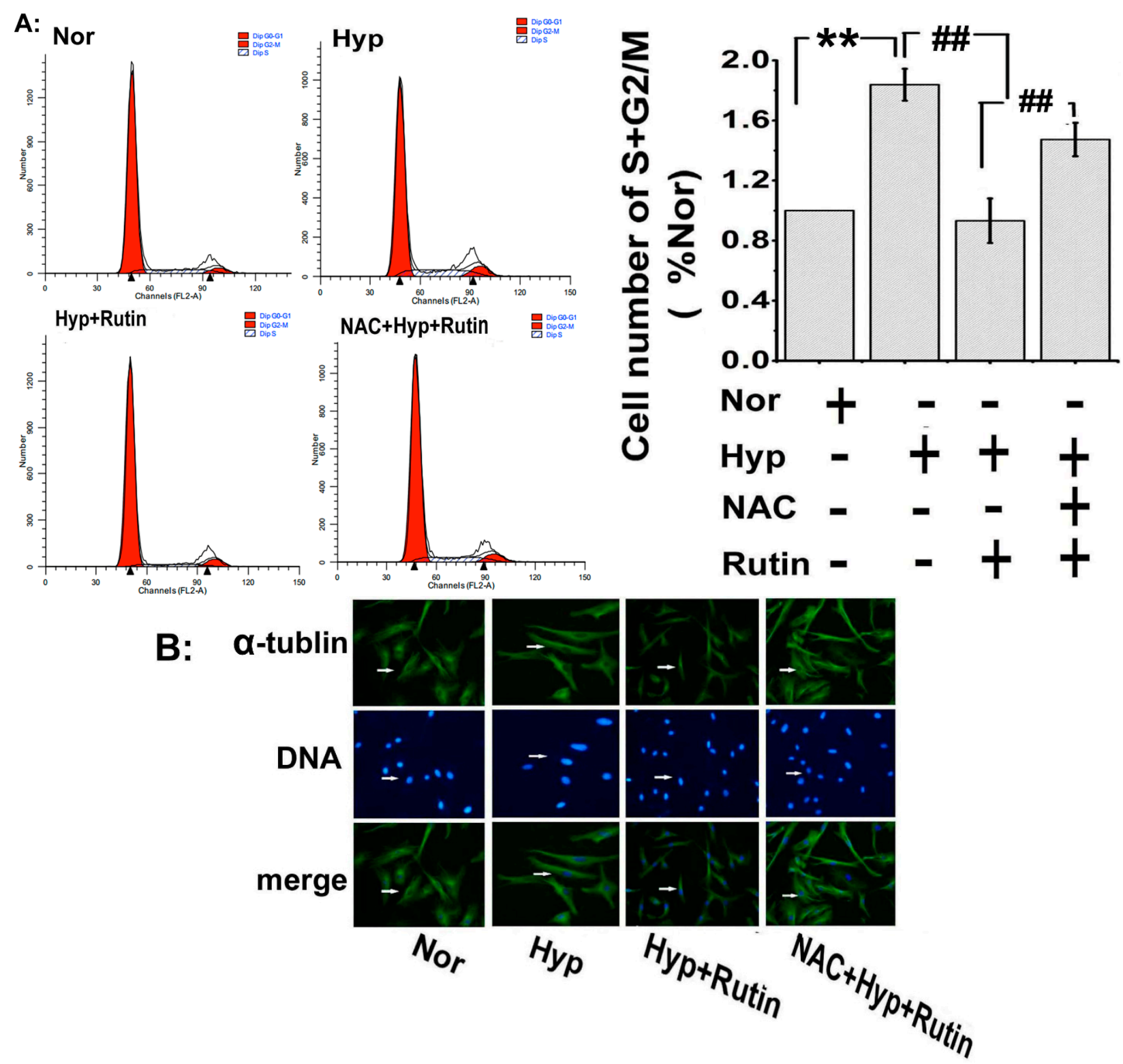


Figure 6. Rutin affected the expression of hypoxia-inducible factor-1 $\alpha$ (HIF-1 $\alpha$ ) when exposed under hypoxia. The upregulated expression of HIF-1 $\alpha$ of PAECs (A) and PASMCs (B) induced by hypoxia was repressed by rutin, and the effect of rutin on hypoxia-induced overexpression of HIF-1 $\alpha$ was alleviated by NAC. All of the values were denoted as mean $\pm \mathrm{SD}$; $* * p<0.01$, versus normal; ${ }^{\#} p<0.01$ versus Hyp. $n=3$. Nor indicates normoxia, Hyp indicates hypoxia.
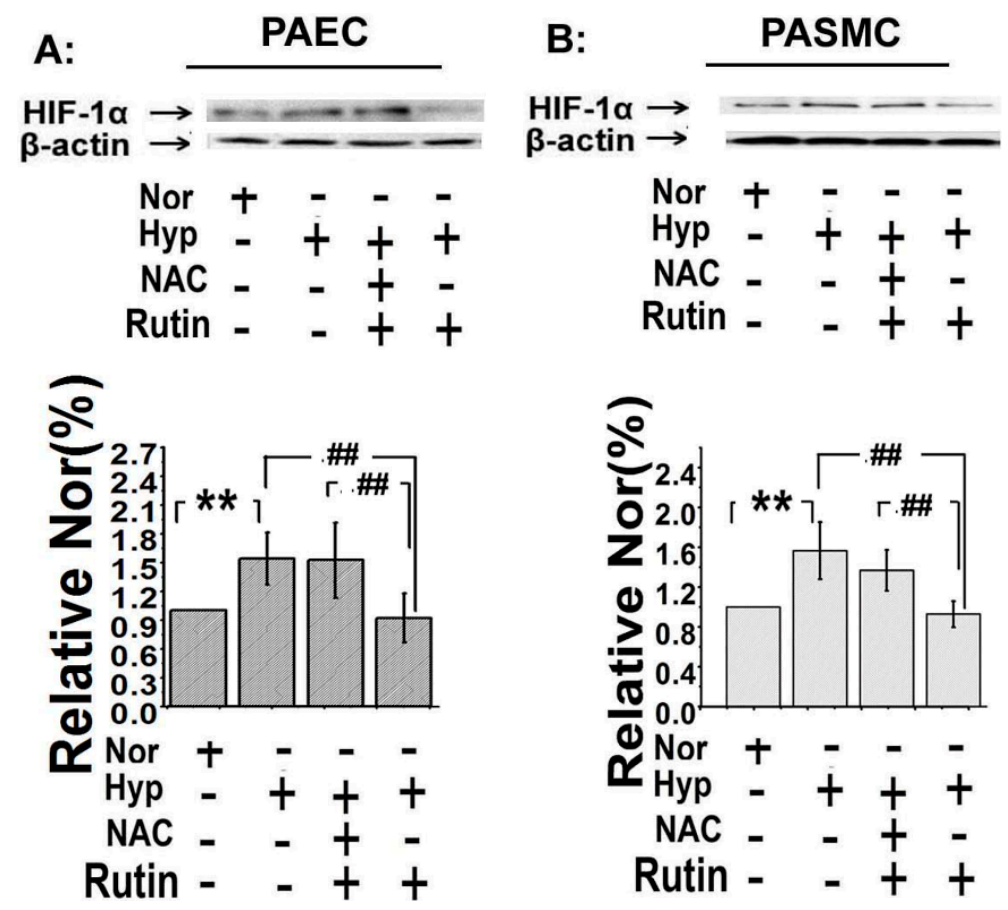

\subsection{Discussion}

In this study, we mainly highlighted two new concepts from our results. First, rutin behaved as an inhibitor of abnormal PA cells proliferation induced by hypoxia by reducing the production of ROS. The main sources of ROS include the mitochondria respiratory chain and NADPH oxidase. Second, the effect of rutin on bovine pulmonary artery cells proliferation was attributed to impairment in the NOX 4 -HIF-1 $\alpha$ pathway.

As reported, hypoxia increased the production of ROS and regulated PA cells proliferation, which is a key component of pulmonary vascular remodeling, leading to pulmonary hypertension [19,28]. Rutin is an antioxidant with many interesting pharmacological effects, such as strengthening and modulating the permeability of the walls of blood vessels [29-31]. However, the role of rutin in reversing the hypoxia-induced abnormal proliferation by reducing ROS still remained elusive; we hypothesized that the effect of rutin on PA cells is relevant to ROS, which might reverse the influence of hypoxia on ROS. In order to test our hypothesis, we used immunofluorescence with $\mathrm{CM}-\mathrm{H}_{2} \mathrm{DCFDA}$ or mitosox to evaluate the production and cell location of ROS under hypoxia. Consistent with previous reports, our data showed hypoxia increased ROS generation, and rutin can block this effect, which verified our concepts. The main sources of ROS regulated by rutin under hypoxic circumstance were the mitochondria respiratory chain and NADPH oxidase. These observations indicated that mtROS contributed to the effect of rutin on PAECs under hypoxia. 
It is well known that the NADPH (nicotinamideadenine dinucleotide phosphate) oxidase family includes seven isoforms. Nox4 is a unique Nox isoform which is described as an oxygen sensor [28,32]. Recent studies using Nox 4 transgenic and knockout mouse models, show a new paradigm is emerging that Nox 4 may be vasculoprotective and upregulation of this Nox isoform may have potential therapeutic benefit in preventing vascular disease [33]. Based on our previous work, we tried to observe the effect of rutin on Nox4 expression and explored the cellular mechanism of this process. Western blot results showed the expression of Nox 4 was increased by hypoxia and this effect could be blocked by rutin These observations demonstrated that rutin decreased the activity of Nox4, reversed the abnormal increase in ROS production induced by hypoxic circumstance, and subsequently contributed to the potent protecting effect during the pathologic process of hypoxia-induced $\mathrm{PAH}$.

Consistent with previous reports [19,31], our data indicated hypoxia stimulated cell cyclins, increased $\alpha$-tubulin polymerization in the nucleus, upregulated expression of PCNA (the proliferating cell nuclear antigen), and led to cell proliferation. The effect of hypoxia on PA cells was blocked by rutin by a ROS-dependent way. These observations elucidated the molecular mechanisms by which rutin modulated the production of ROS, which may lead to insights into defining the role of rutin in PA cell proliferation occurring in hypoxic pulmonary remodeling.

In mammals, the primary transcriptional response to hypoxic stress was mediated by the activation of HIF-1 $\alpha[19,27]$. In this study, we found the overexpression of HIF-1 $\alpha$ under hypoxia was alleviated by rutin, implying that rutin may benefit the proliferation of pulmonary artery cells through inhibited HIF- $1 \alpha$ activity.

\section{Experimental Section}

\subsection{Materials}

Rutin was obtained from the Chinese National Institutes of Food and Drug Control through common commercial sources. NAC ( $N$-acetyl-L-cysteine), APO (apocynin), and RE (rotenone) were purchased from Sigma-Aldrich Corporation (St. Louis, MO, USA). Antibodies against Nox4, PCNA, HIF-1 $\alpha$ and $\alpha$-tubulin were purchased from Santa Cruz Biotechnology Inc. (Santa Cruz, Texas, TX, USA). Reactive Oxygen Species (ROS) detection reagents $\mathrm{CM}-\mathrm{H}_{2}$ DCFDA and mitosox red mitochondrial superoxide indicator were obtained from Life Technologies (AB \& Invitrogen, Carlsbad, CA, USA). All other reagents were also from common commercial sources.

\subsection{Cell Isolation and Culture}

PAECs and PASMCs were prepared from pulmonary arterial of calf lungs, obtained from a local slaughterhouse and approved by the Harbin Medical University Ethical Committee of Laboratory Animals. The arteries were gently slit open and the innermost layer scraped with a surgical blade to obtain the endothelial cells, after which the arteries were cut into small pieces and the smooth muscle layer affixed tightly to the culture dish. The arterial fractions were then covered with Dulbecco's modified eagle's medium (DMEM) with $20 \%$ fetal bovine serum (FBS). After the PASMCs were taken out the tissue fractions were then lifted out. The purity and identity of the PAECs and PASMCs were confirmed by anti-CD31 (Santa Cruz), and anti- $\alpha$-actin, respectively. 


\subsection{Immunofluorescence}

After pre-treatment with the ROS inhibitor (apocynin or rotenone) for half an hour, the cells were treated with rutin for $2 \mathrm{~h}$, then washed three times with the indicated buffer solution, before incubation with ROS detection reagents or mitosox for the appropriate time. Afterwards, the cells were washed with buffer solution three times as per instructions. The fluorescence intensity was photographed and analyzed by confocal laser scanning microscope (CLSM).

\subsection{Scratch-Wound Assay}

ECs were cultivated in six-well culture plates and scratched with pipette tips. Then the cells were added with rutin or NAC plus rutin in 5\% FBS of DMEM under hypoxia at the indicated concentrations. The same field was photographed at $0 \mathrm{~h}$ and $8 \mathrm{~h}$. The area of migration was calculated by counting the migration using Image Pro-Plus 6.0.

\subsection{Immunocytochemistry}

PASMCs were cultured on a poly-L-lysine-coated cover glass (15 mm diameter) and washed with buffer solution, followed by fixation with $4 \%$ paraformaldehyde at room temperature for $15 \mathrm{~min}$. After permeabilization with $0.01 \%$ Triton $\mathrm{X}-100$ for $10 \mathrm{~min}$, the cells were blocked with $3 \%$ normal bovine serum at $37{ }^{\circ} \mathrm{C}$ for $30 \mathrm{~min}$, then incubated with anti- $\alpha$-tubulin primary antibodies $(1: 50)$ in PBS at $4{ }^{\circ} \mathrm{C}$ overnight. Washed with PBS three times, the cells were incubated with FITC-conjugated secondary antibody (1:100) and Hoechst at $37^{\circ} \mathrm{C}$ for $2 \mathrm{~h}$. The images were captured and analyzed by CLSM.

\subsection{Western Blot Analysis}

After pre-treatment with rutin $(0.1 \mu \mathrm{mol} / \mathrm{L})$, or NAC $(5 \mu \mathrm{mol} / \mathrm{L})$ plus rutin in DMEM with $5 \%$ FBS for $24 \mathrm{~h}$, the cells were washed with PBS buffer three times. The lysates were prepared with RIPA lysis buffer. The protein concentration was measured by the bicinchoninic acid protein assay (Pierce, Rockford, IL, USA), based on bovine serum albumin (BSA) standard. The proteins were separated by $10 \%$ SDS-PAGE, and electro transferred to a nitrocellulose membrane (Merck Millipore, Beijing, China). Membranes were blocked with $5 \%$ milk in TBST for $3 \mathrm{~h}$ at $4{ }^{\circ} \mathrm{C}$, followed by incubating with primary antibodies against Nox4, PCNA, HIF-1 $\alpha$ (all 1:200 in 5\% BSA) at $4{ }^{\circ} \mathrm{C}$ overnight. The treated membranes were then exposed to horseradish peroxidase-conjugated secondary antibody $(1: 10,000$, Santa Cruz Biotechnology) for half an hour and enhanced with chemiluminescence reagents.

\subsection{MTT Assay}

PASMCs were cultured in 96-well culture plates before exposing to different reagents at the indicated concentrations for $24 \mathrm{~h}$. After adding 0.5\% 3-(4,5-dimethylthiazol-2-yl)-2,5-diphenyl-tetrazolium bromide (MTT), the survival cells formed a blue formazan dye derived from MTT. The reaction was terminated by DMSO and evaluated by spectrophotometric absorbance at $540 \mathrm{~nm}$. 


\subsection{Flow Cytometry}

After treating with different reagents at normal concentrations for $24 \mathrm{~h}$, the cultured cells were then washed with PBS, and collected before being fixed with $75 \%$ ethanol for another $24 \mathrm{~h}$ at $4{ }^{\circ} \mathrm{C}$. After incubation in $0.5 \mathrm{~mL}$ PBS $\left(10 \mu \mathrm{g} / \mathrm{mL}\right.$ Rnase A and $100 \mu \mathrm{g} / \mathrm{mL}$ PI) for $30 \mathrm{~min}$ at $37^{\circ} \mathrm{C}$ away from light, the immobilized cell samples were then measured for DNA fluorescence using a BD FACSCalibur Flow Cytometer (Bedford, MA, USA). The DNA content at each phase of the cell cycle was recorded.

\subsection{Statistics}

Data are presented as mean $\pm \mathrm{SD}$. For comparisons between two experimental groups, the student's paired t-test was used. Significant level was set at $p<0.05$. Statistical analysis was performed by Statistical Product and Service Solutions version 13.0 (IBM SPSS, Beijing, China).

\section{Conclusions}

We hypothesized that the natural product, rutin, was capable of regulating the unusual expression of mitochondrial Nox4 induced by hypoxia and influencing the production of ROS, which subsequently affects the proliferation and remodeling of the pulmonary artery; our observations then provided evidence to support our hypothesis.

In conclusion, rutin possesses specific properties in regulating abnormal changes during the pathological process and could serve as a new target in the treatment of hypoxic pulmonary hypertension. Further studies are required to explore the mechanism of the cell signaling pathways with regard to the antioxidant mechanism process of rutin on hypoxia-induced pulmonary arterial cell proliferation.

\section{Acknowledgments}

We are grateful for the financial support from the National Natural Science Foundation of China (grant number 31100835); National Foundation for Post-doctoral Scholars (2013M531066); Heilongjiang Province Foundation for Post-doctoral Scholars (LBH-Z11059); Fund of Heilongjiang Province Education Department (No. 12511316).

\section{Author Contributions}

Qian Li and Xiaodong Zheng designed the experiments. Qian Li, Yanli Qiu, Min Mao, Jinying Lv, Lixin Zhang, Shuzhen Li, Xia Li performed the experiment. Qian Li, Yanli Qiu wrote the paper, and Xiaodong Zheng revised the paper. All authors discussed the results and commented on the manuscript.

\section{Conflicts of Interest}

The authors declare no conflict of interest. 


\section{References}

1. Parent, F.; Bachir, D.; Inamo, J.; Lionnet, F.; Driss, F.; Loko, G.; Habibi, A.; Bennani, S.; Savale, L.; Adnot, S. A hemodynamic study of pulmonary hypertension in sickle cell disease. N. Engl. J. Med. 2011, 365, 44-53.

2. Tabima, D.M.; Frizzell, S.; Gladwin, M.T. Reactive oxygen and nitrogen species in pulmonary hypertension. Free Radic. Biol. Med. 2012, 52, 1970-1986.

3. Voelkel, N.F.; Tuder, R.M. Hypoxia-induced pulmonary vascular remodeling: A model for what human disease? J. Clin. Invest. 2000, 106, 733-738.

4. Stenmark, K.R.; Meyrick, B.; Galie, N.; Mooi, W.J.; McMurtry, I.F. Animal models of pulmonary arterial hypertension: The hope for etiological discovery and pharmacological cure. Am. J. Physiol. Lung Cell. Mol. Physiol. 2009, 297, L1013-L1032.

5. Mandegar, M.; Fung, Y.C.; Huang, W.; Remillard, C.V.; Rubin, L.J.; Yuan, J.X. Cellular and molecular mechanisms of pulmonary vascular remodeling: Role in the development of pulmonary hypertension. Microvasc. Res. 2004, 68, 75-103.

6. Morrell, N.W.; Adnot, S.; Archer, S.L.; Dupuis, J.; Jones, P.L.; MacLean, M.R.; McMurtry, I.F.; Stenmark, K.R.; Thistlethwaite, P.A.; Weissmann, N.; et al. Cellular and molecular basis of pulmonary arterial hypertension. J. Am. Coll. Cardiol. 2009, 54 (Suppl. 1), S20-S31.

7. Ogura, S.; Shimosawa, T.; Mu, S.; Sonobe, T.; Kawakami-Mori, F.; Wang, H.; Uetake, Y.; Yoshida, K.; Yatomi, Y.; Shirai, M.; et al. Oxidative stress augments pulmonary hypertension in chronically hypoxic mice overexpressing the oxidized LDL receptor. Am. J. Physiol. Heart Circ. Physiol. 2013, 305, H155-H162.

8. Fan, Y.F.; Zhang, R.; Jiang, X.; Wen, L.; Wu, D.C.; Liu, D.; Yuan, P.; Wang, Y.L.; Jing, Z.C. The phosphodiesterase-5 inhibitor vardenafil reduces oxidative stress while reversing pulmonary arterial hypertension. Cardiovasc. Res. 2013, 99, 395-403.

9. Konduri, G.G.; Bakhutashvili, I.; Eis, A.; Pritchard, K., Jr. Oxidant stress from uncoupled nitric oxide synthase impairs vasodilation in fetal lambs with persistent pulmonary hypertension. Am. J. Physiol. Heart Circ. Physiol. 2007, 292, H1812-H1820.

10. Waypa, G.B.; Marks, J.D.; Mack, M.M.; Boriboun, C.; Mungai, P.T.; Schumacker, P.T. Mitochondrial reactive oxygen species trigger calcium increases during hypoxia in pulmonary arterial myocytes. Circ. Res. 2002, 91, 719-726.

11. Wedgwood, S.; Dettman, R.W.; Black, S.M. ET-1 stimulates pulmonary arterial smooth muscle cell proliferation via induction of reactive oxygen species. Am. J. Physiol. Lung Cell. Mol. Physiol. 2001, 281, L1058-L1067.

12. Ray, P.D.; Huang, B.W.; Tsuji, Y. Reactive oxygen species (ROS) homeostasis and redox regulation in cellular signaling. Cell. Signal. 2012, 24, 981-990.

13. Basuroy, S.; Tcheranova, D.; Bhattacharya, S.; Leffler, C.W.; Parfenova, H. Nox4 NADPH oxidase-derived reactive oxygen species, via endogenous carbon monoxide, promote survival of brain endothelial cells during TNF- $\alpha$-induced apoptosis. Am. J. Physiol. Cell Physiol. 2011, 300, C256-C265. 
14. Kuhr, F.K.; Smith, K.A.; Song, M.Y.; Levitan, I.; Yuan, J.X. New mechanisms of pulmonary arterial hypertension: Role of $\mathrm{Ca}^{2+}$ signaling. Am. J. Physiol. Heart Circ. Physiol. 2012, 302, H1546-H1562.

15. Shimoda, L.A.; Laurie, S.S. Vascular remodeling in pulmonary hypertension. J. Mol. Med. 2013, 91, 297-309.

16. Kelman, Z. PCNA: Structure, functions and interactions. Oncogene 1997, 14, 629-640.

17. Strzalka, W.; Ziemienowicz, A. Proliferating cell nuclear antigen (PCNA): A key factor in DNA replication and cell cycle regulation. Ann. Bot. 2011, 107, 1127-1140.

18. Zhang, D.; Ma, C.; Li, S.; Ran, Y.; Chen, J.; Lu, P.; Shi, S.; Zhu, D. Effect of Mitofusin 2 on smooth muscle cells proliferation in hypoxic pulmonary hypertension. Microvasc. Res. 2012, 84, 286-296.

19. Semenza, G.L. Hypoxia-inducible factors in physiology and medicine. Cell 2012, 148, 399-408.

20. Shimoda, L.A.; Semenza, G.L. HIF and the lung: Role of hypoxia-inducible factors in pulmonary development and disease. Am. J. Respir. Crit. Care Med. 2011, 183, 152-156.

21. La Casa, C.; Villegas, I.; Alarcón de la Lastra, C.; Motilva, V.; Martin Calero, M.J. Evidence for protective and antioxidant properties of rutin, a natural flavone, against ethanol induced gastric lesions. J. Ethnopharmacol. 2000, 71, 45-53.

22. Kamalakkannan, N.; Prince, P.S. Antihyperglycaemic and antioxidant effect of rutin, a polyphenolic flavonoid, in streptozotocin-induced diabetic wistar rats. Basic Clin. Pharmacol. Toxicol. 2006, 98, 97-103.

23. Li, Q.; Niu, S.; Wang, R.; Li, Y.; Zhang, R.; Zhu, D. Mechanisms that underlie the induction of vasodilation in pulmonary artery by rutin. Int. Angiol. 2012, 31, 557-564.

24. Xiao, G.F.; Xu, S.H.; Chao, Y.; Xie, L.D.; Xu, C.S.; Wang, H.J. PPARdelta activation inhibits homocysteine-induced p22(phox) expression in EA.hy926 cells through reactive oxygen species/p38MAPK pathway. Eur. J. Pharmacol. 2014, 727, 29-34.

25. Wedgwood, S.; Lakshminrusimha, S.; Czech, L.; Schumacker, P.T.; Steinhorn, R.H. Increased p22(phox)/Nox4 expression is involved in remodeling through hydrogen peroxide signaling in experimental persistent pulmonary hypertension of the newborn. Antioxid. Redox Signal. 2013, 18, 1765-1776.

26. Stones, R.; Benoist, D.; Peckham, M.; White, E. Microtubule proliferation in right ventricular myocytes of rats with monocrotaline-induced pulmonary hypertension. J. Mol. Cell. Cardiol. 2013, 56, 91-96.

27. Wolin, M.S. Novel role for the regulation of mitochondrial fission by hypoxia inducible factor- $1 \alpha$ in the control of smooth muscle remodeling and progression of pulmonary hypertension. Circ. Res. 2012, 110, 1395-1397.

28. Yang, X.; Sheares, K.K.; Davie, N.; Upton, P.D.; Taylor, G.W.; Horsley, J.; Wharton, J.; Morrell, N.W. Hypoxic induction of cox-2 regulates proliferation of human pulmonary artery smooth muscle cells. Am. J. Respir. Cell Mol. Biol. 2002, 27, 688-696.

29. Khan, R.A.; Khan, M.R.; Sahreen, S. Protective effects of rutin against potassium bromate induced nephrotoxicity in rats. BMC Complement. Altern. Med. 2012, 12, doi:10.1186/1472-6882-12-204. 
30. Azevedo, M.I.; Pereira, A.F.; Nogueira, R.B.; Rolim, F.E.; Brito, G.A.; Wong, D.V.; Lima-Junior, R.C.; de Albuquerque Ribeiro, R.; Vale, M.L. The antioxidant effects of the flavonoids rutin and quercetin inhibit oxaliplatin-induced chronic painful peripheral neuropathy. Mol. Pain 2013, 9, doi:10.1186/1744-8069-9-53.

31. Panchal, S.K.; Poudyal, H.; Arumugam, T.V.; Brown, L. Rutin attenuates metabolic changes, nonalcoholic steatohepatitis, and cardiovascular remodeling in high-carbohydrate, high-fat diet-fed rats. J. Nutr. 2011, 141, 1062-1069.

32. Montezano, A.C.; Burger, D.; Ceravolo, G.S.; Yusuf, H.; Montero, M.; Touyz, R.M. Novel Nox homologues in the vasculature: Focusing on Nox4 and Nox5. Clin. Sci. 2011, 120, 131-141.

33. Schröder, K.; Zhang, M.; Benkhoff, S.; Mieth, A.; Pliquett, R.; Kosowski, J.; Kruse, C.; Lüdike, P.; Michaelis, U.R.; Weissmann, N. Nox4 is a protective reactive oxygen species generating vascular NADPH oxidase. Circ. Res. 2012, 110, 1217-1225.

Sample Availability: Samples of the compounds are available from the authors.

(C) 2014 by the authors; licensee MDPI, Basel, Switzerland. This article is an open access article distributed under the terms and conditions of the Creative Commons Attribution license (http://creativecommons.org/licenses/by/4.0/). 